\section{INCIDENCE AND OUTCOME OF GROUP B STREPTOCOCCAL SEPSIS IN INFANTS IN SWITZERLAND}

\author{
Eric Giannoni, $M D,{ }^{*}+$ Christoph Berger, $M D,+$ \\ Martin Stocker, MD, \& Philipp Agyeman, MD, \\ Klara M. Posfay-Barbe, MD, \| Ulrich Heininger, MD, ** \\ Gabriel Konetzny, MD, $\uparrow$ Anita Niederer-Loher, MD, + t \\ Christian Kahlert, MD, $\$$ Alex Donas, $M D$, $\$$ \\ Antonio Leone, MD, $\S \S$ Paul Hasters, MD, $\$ \S$ \\ Christa Relly, MD, \$ Walter Baer, MD, 19 Christoph Aebi, MD, 9 \\ and Luregn J. Schlapbach, MD, $\mathbb{9}\|\| \|^{* *}$ for the Swiss Pediatric \\ Sepsis Study Group
}

\begin{abstract}
The incidence and outcome of group B streptococcal (GBS) sepsis were assessed prospectively between September 2011 and February 2015 in all tertiary care pediatric hospitals of Switzerland. We describe a low incidence of GBS early-onset sepsis (0.12/1000 livebirths) and a predominance of GBS late-onset sepsis (0.36/1000 livebirths), a pattern that has not been reported in other countries.
\end{abstract}

Key Words: Streptococcus agalactiae, group B streptococcus, early-onset, late-onset neonatal sepsis, meningitis

Accepted for publication September 4, 2015.

From the *Service of Neonatology, Lausanne University Hospital, University of Lausanne, Lausanne, Switzerland; †Infectious Diseases Service, Lausanne University Hospital, University of Lausanne, Lausanne, Switzerland; $\$$ Division of Infectious Diseases, Children's Research Center, University Children's Hospital Zurich, Zurich, Switzerland; §Department of Pediatrics, Children's Hospital Lucerne, Lucerne, Switzerland; 9 Department of Pediatrics, Inselspital, University of Bern, Bern, Switzerland; \#Pediatric Infectious Diseases Unit, Children's Hospital of Geneva, University Hospitals of Geneva, Geneva, Switzerland; **Infectious Diseases and Vaccinology, University Children's Hospital Basel, Basel, Switzerland; ††Department of Pediatrics, Children's Hospital Aarau, Aarau, Switzerland; +\$Department of Infectious Diseases, Children’s Hospital of Eastern Switzerland St. Gallen, St. Gallen, Switzerland; §§Department of Neonatology, University Hospital Zurich, Zurich, Switzerland; q币Department of Pediatrics, Children's Hospital Chur, Chur, Switzerland; \|\| Paediatric Critical Care Research Group, Mater Research Institute, University of Queensland, Brisbane, Australia; and ***Paediatric Intensive Care Unit, Lady Cilento Children's Hospital, Children's Health Queensland, Brisbane, Australia

Supported by grants from the Swiss National Science Foundation (342730_153158/1), the Swiss Society of Intensive Care, the Bangerter Foundation, the Vinetum and Borer Foundation and the Foundation for the Health of Children and Adolescents. The funding sources did not have any role in the design of the study, nor in the analyses, nor in the writing of the manuscript or decision to submit it for publication. The authors have no conflicts of interest to disclose.

Address for correspondence: Eric Giannoni, MD, Service de Néonatologie, Avenue Pierre Decker 2, Centre Hospitalier Universitaire Vaudois, CH-1011 Lausanne, Switzerland. E-mail: Eric.Giannoni@chuv.ch.

Copyright (C) 2015 Wolters Kluwer Health, Inc. All rights reserved. DOI: $10.1097 / \mathrm{INF} .0000000000000974$

$S_{a}^{n}$ eptococcus agalactiae or group B streptococcus (GBS) is among the most frequent pathogens causing sepsis during infancy. ${ }^{1}$ Because of differences in pathogenesis, clinical presentation and outcome, GBS disease is categorized into early-onset sepsis (EOS) and late-onset sepsis (LOS). After the implementation of intrapartum antibiotic prophylaxis (IAP), surveillance studies have reported a $50 \%-85 \%$ reduction of GBS-EOS, whereas the incidence of GBS-LOS has remained stable. ${ }^{2}$ However, recent studies performed in the United States, Europe and Australia indicate that, despite the marked reduction associated with the recommendations for IAP, the incidence of GBS-EOS remains similar ${ }^{3,4}$ or higher ${ }^{1,5,6}$ compared with the incidence of GBS-LOS. Yet, important geographical differences exist in the epidemiology of GBS disease and its prevention strategies. ${ }^{1,6}$ In this study, we assessed the incidence, clinical features and outcome of GBS sepsis over a 3.5-year period in Switzerland, a country where IAP is administered on the basis of maternal antepartum screening results, following the recommendations of the Centers for Disease Control and Prevention. ${ }^{3}$

\section{PATIENTS AND METHODS}

The Swiss Pediatric Sepsis Study prospectively investigates the epidemiology of blood culture-proven sepsis in children in all 10 tertiary care pediatric hospitals of Switzerland. These centers host all neonatal and pediatric intensive care units of the country. Infants from birth to $<1$ year of age were eligible for this study if they developed bacteremia between September 2011 and February 2015, in the presence of a systemic inflammatory response syndrome, as defined by the consensus statement. ${ }^{7}$ Contaminations were excluded. Positive blood cultures were identified automatically by microbiology laboratory notification systems, and systemic inflammatory response was confirmed prospectively by clinicians. The study was approved by the ethics committees of all participating centers. Data on demographics, perinatal risk factors, infection focus, severity and outcome were recorded prospectively. EOS was defined as infection presenting $<7$ days of life, and LOS was defined as infection presenting $\geq 7$ days. Each episode of sepsis was considered as a single case. The incidence of GBS sepsis was calculated for infants born at the study centers. We also calculated the national incidence of GBS sepsis, estimating that the participating centers admitted $75 \%$ of all infants treated for sepsis in Switzerland. The number of livebirths in Switzerland was obtained from the Swiss Federal Statistical Office.

Statistical analyses were performed using PRISM (GraphPad Software Inc., La Jolla, CA). Baseline clinical characteristics were described by showing the median and the interquartile range (IQR) for continuous variables and numbers and percentages for categorical variables. Comparisons of continuous and categorical variables between cases of EOS and LOS were performed using 2-tailed $t$ tests and Pearson $\chi^{2}$ tests.

\section{RESULTS}

Five hundred thirty-nine episodes of blood culture-proven sepsis were diagnosed in infants during a 3.5-year period. GBS was the third most common pathogen with $14 \%(n=74)$ of episodes. In comparison, Escherichia coli and coagulase-negative staphylococci were identified in $23 \%(n=122)$ and $19 \%(n=103)$ of sepsis episodes, respectively. Among cases of GBS sepsis, 30\% $(n=22)$ presented as EOS and 70\% $(n=52)$ as LOS (Table 1). Four patients had recurrent GBS sepsis: 3 patients had 2 episodes of GBS-LOS and 1 patient had both GBS-EOS and GBS-LOS.

The total number of livebirths was 69,118 in the study centers and 289,543 in the whole country. The incidence of GBS-EOS and GBS-LOS was 0.12 [95\% confidence interval (CI): 0.05-0.23] and $0.36 / 1000$ livebirths (95\% CI: $0.23-0.53$ ), respectively, for infants born at the study centers. The estimated national incidence of GBS-EOS and GBS-LOS in Switzerland was $0.10(95 \% \mathrm{CI}$ : $0.06-0.15$ ) and $0.24 / 1000$ livebirths (95\% CI: $0.18-0.31$ ), respectively, which is not significantly different from the incidence at the study centers $(P=0.75$ for EOS and 0.09 for LOS). In infants born $<37$ weeks of gestation, the estimated national incidence of GBSEOS and LOS was 0.36/1000 livebirths (95\% CI: 0.16-0.78) and 1.01/1000 livebirths (95\% CI: 0.63-1.62), respectively. For infants born with a birth weight $<1500 \mathrm{~g}$, the estimated incidence of GBSEOS and LOS was 1.04/1000 livebirths (95\% CI: $0.35-3.04)$ and 3.80/1000 livebirths (95\% CI: 2.12-6.79), respectively.

Among patients with GBS-EOS, 73\% (16/22) presented with clinical signs on the first day of life and $91 \%(20 / 22)$ presented 
TABLE 1. Baseline Demographics and Outcomes of Blood Culture-proven Group B Streptococcal Sepsis in Infants Admitted to the 10 Tertiary Care Pediatric Hospitals of Switzerland

\begin{tabular}{|c|c|c|c|}
\hline & Early-onset Sepsis & Late-onset Sepsis & $P$ Value \\
\hline Number of cases $(\%)$ & $22(29)$ & $52(70)$ & \\
\hline Incidence in inborns $*$ per 1000 livebirths $(95 \% \mathrm{CI})$ & $0.12(0.05-0.23)$ & $0.36(0.23-0.53)$ & 0.003 \\
\hline Estimated incidence per 1000 livebirths (95\% CI) & $0.10(0.06-0.15)$ & $0.24(0.18-0.31)$ & $<0.001$ \\
\hline \multicolumn{4}{|l|}{ Maternal GBS status, $\mathrm{n}(\%)$} \\
\hline Positive & $8(36)$ & $19(37)$ & 0.56 \\
\hline Negative & $10(45)$ & $28(54)$ & \\
\hline Unknown & $4(18)$ & $5(10)$ & \\
\hline Intrapartum antibiotic prophylaxis, $\mathrm{n}(\%)$ & $5(23)$ & $14(27)$ & 0.71 \\
\hline Male gender, n (\%) & $9(41)$ & $29(56)$ & 0.24 \\
\hline Median gestational age at birth, wk (IQR) & $39(35-41)$ & $39(33-40)$ & 0.49 \\
\hline Prematurity, n (\%) & $6(27)$ & $17(33)$ & 0.65 \\
\hline Median birth weight, g (IQR) & $3150(2190-3510)$ & $3000(2230-3330)$ & 0.37 \\
\hline Median age at presentation, $d(I Q R)$ & $0(0-0)$ & $34(18-58)$ & \\
\hline Median time to positivity of blood cultures, $\mathrm{h}$ (IQR) & $15.3(8.5-20.2)$ & $12.0(9.4-28.6)$ & 0.34 \\
\hline Mortality, n (\%) & $2(10)$ & 0 & \\
\hline Septic shock, $\dagger \mathrm{n}(\%)$ & $6(27)$ & $3(6)$ & 0.009 \\
\hline Noninvasive ventilation, n (\%) & $8(36)$ & $8(15)$ & 0.04 \\
\hline Invasive ventilation, $\mathrm{n}(\%)$ & $8(36)$ & $9(17)$ & 0.04 \\
\hline \multicolumn{4}{|l|}{ Focus of infection, $\mathrm{n}(\%)$} \\
\hline Bacteremia without a focus & $17(77)$ & $35(67)$ & 0.39 \\
\hline Meningitis & $4(18)$ & $9(17)$ & 0.93 \\
\hline Pneumonia & $1(5)$ & $2(4)$ & 0.89 \\
\hline Cellulitis & 0 & $5(10)$ & \\
\hline
\end{tabular}

*Includes only infants born at the 10 participating centers.

$\dagger$ As defined in the study by Goldstein et al. ${ }^{\text {? }}$

within the first 2 days of life. For LOS, the median age at clinical presentation was 34 days (IQR: 18-58). Of note, $12 \%$ (6 of 52) of infants with GBS-LOS presented beyond 90 days of age, between 91 and 202 days. In our entire cohort of children younger than 17 years with blood culture-proven sepsis, no patient presented with GBS sepsis at an age $>202$ days.

The median gestational age at birth was 39 1/7 weeks (IQR: 35-41) for patients with EOS and 39 weeks (IQR: 33-40) for those with LOS. The median birth weight was $3150 \mathrm{~g}$ (IQR: 2190-3510) for EOS and $3000 \mathrm{~g}$ (IQR: 2230-3330) for LOS. Maternal GBS status was unknown to the obstetrical team at the time of birth in $12 \%$ ( 9 of 74) of cases of GBS sepsis. Of the mothers who were screened for GBS, $42 \%$ (27 of 65 ) were positive, but only $48 \%$ (13 of 27 ) of them received IAP. Eleven (21\%) episodes of GBS-LOS occurred after $>2$ days of hospitalization. All (11 of 11) infants with hospitalacquired GBS-LOS were preterm, whereas $15 \%$ (6 of 41 ) of infants with community-acquired GBS-LOS were preterm.

In $70 \%$ of infants $(\mathrm{n}=52)$, GBS sepsis presented as bacteremia without a focus. All GBS strains were susceptible to penicillin. Patients with GBS-EOS were more likely to present with septic shock than patients with GBS-LOS, and they more frequently required noninvasive and/or invasive ventilation. Two (3\%) infants with GBS sepsis died: an infant born at 29 6/7 weeks of gestation died on the first day of life because of GBS septic shock; a term infant with GBS-EOS died at 4 weeks of age because of complications of a congenital heart disease.

\section{DISCUSSION}

We report on prospective national surveillance of GBS blood culture-proven sepsis in Switzerland. This study confirms that GBS remains among the top 3 causes of sepsis in infants, together with Escherichia coli and coagulase-negative staphylococci. Our results show a strong predominance of GBS-LOS over GBS-EOS in Switzerland. This pattern, which has not been described in other countries, is because of a particularly low incidence of GBS-EOS in
Switzerland. Indeed, the incidence of GBS-EOS in our cohort is 2- to 4-fold lower than the incidence reported in other high-income countries, whereas the incidence of GBS-LOS is in the range of previous studies performed in other European countries, Australia and North America. ${ }^{1,4-6,8}$

The reason for a low incidence of GBS-EOS in Switzerland is unclear. National data on the rate of rectovaginal GBS carriage in pregnant women and IAP are not available in Switzerland. However, low rates of colonization are unlikely as GBS carriage was identified in $16 \%$ of pregnant women in a Swiss hospital, which is comparable with reports from other countries. ${ }^{9}$ In all centers participating in our study, IAP was administered based on routine screening of pregnant women for GBS, as recommended by the Centers for Disease Control and Prevention. ${ }^{3}$ Yet, the proportion of all GBS-positive mothers who received IAP was not recorded in our study. Only half of the infants with GBS sepsis who were born to GBS-positive mothers were exposed to IAP. This shows that missed opportunities for prevention still exist. Reinforcing the implementation of maternal GBS screening and improving the compliance to administration of IAP could lead to a further decrease in the incidence of GBS-EOS in Switzerland.

In agreement with the previous studies, the majority of infants with EOS presented within the first 24 hours and almost all $(91 \%)$ within the first 2 days of life. Interestingly, $12 \%$ of the GBS-LOS cases presented after 90 days of age, indicating that the vulnerability to invasive GBS disease remains high throughout the first year of life.

With a $1.4 \%$ direct GBS-related fatality rate, the overall mortality was lower in our cohort compared with other studies that reported a $4.6 \%$ fatality rate. ${ }^{1}$ However, disease severity was considerable with $23 \%$ of cases requiring invasive ventilation and $12 \%$ presenting with septic shock. Disease severity was greater in EOS compared with LOS, which may reflect an exposure to a higher bacterial load perinatally, less-efficient host defenses and/or a lower functional reserve of vital organs during adaptation to extrauterine life. 
A limitation of this study is related to the fact that we included only the episodes of GBS disease with positive blood cultures. Previous reports indicate that $13 \%-38 \%$ of infants with GBS meningitis have negative blood cultures. Therefore, our study may underestimate the total burden of GBS invasive disease. ${ }^{4,5,8}$ A second limitation is that the surveillance, which was based on the 10 main pediatric hospitals of Switzerland, may have missed some cases with milder clinical presentation that were treated in regional hospitals. However, because all neonatal and pediatric intensive care units of Switzerland participated in this study, we can assume that all severe cases were captured. The estimated national incidence was not different from the incidence of GBS sepsis calculated for the infants born at the participating centers, suggesting that our study is providing a robust estimate of the epidemiology of GBS sepsis in Switzerland. The long-term consequences of GBS sepsis were not evaluated in our cohort, but it is well known that neonatal sepsis and meningitis are associated with adverse neurodevelopmental outcomes. ${ }^{10}$

In conclusion, we describe a low incidence of GBS-EOS in Switzerland, with a predominance of GBS-LOS. These findings confirm that the incidence of GBS sepsis varies considerably between high-income countries. ${ }^{1}$ Our results highlight the need for epidemiological surveillance of GBS disease and warrant for caution when extrapolating data to other populations. Despite large efforts to reduce the impact of GBS disease, GBS continues to cause significant burden on neonatal and infant health. Better strategies are required for the prevention of both early- and late-onset GBS disease.

\section{REFERENCES}

1. Edmond KM, Kortsalioudaki C, Scott S, et al. Group B streptococcal disease in infants aged younger than 3 months: systematic review and metaanalysis. Lancet. 2012;379:547-556.
2. Moore MR, Schrag SJ, Schuchat A. Effects of intrapartum antimicrobial prophylaxis for prevention of group-B-streptococcal disease on the incidence and ecology of early-onset neonatal sepsis. Lancet Infect Dis. 2003;3:201-213

3. Verani JR, McGee L, Schrag SJ; Division of Bacterial Diseases, National Center for Immunization and Respiratory Diseases, Centers for Disease Control and Prevention (CDC). Prevention of perinatal group B streptococcal disease-revised guidelines from CDC, 2010. MMWR Recomm Rep. 2010;59:1-36

4. Jordan HT, Farley MM, Craig A, et al; Active Bacterial Core Surveillance (ABCs)/Emerging Infections Program Network, CDC. Revisiting the need for vaccine prevention of late-onset neonatal group B streptococcal disease: a multistate, population-based analysis. Pediatr Infect Dis J. 2008;27:1057-1064.

5. Fluegge K, Siedler A, Heinrich B, et al; German Pediatric Surveillance Unit Study Group. Incidence and clinical presentation of invasive neonatal group B streptococcal infections in Germany. Pediatrics. 2006;117:e1139-e1145.

6. Bekker V, Bijlsma MW, van de Beek D, et al. Incidence of invasive group B streptococcal disease and pathogen genotype distribution in newborn babies in the Netherlands over 25 years: a nationwide surveillance study. Lancet Infect Dis. 2014;14:1083-1089.

7. Goldstein B, Giroir B, Randolph A; International Consensus Conference on Pediatric Sepsis. International pediatric sepsis consensus conference: definitions for sepsis and organ dysfunction in pediatrics. Pediatr Crit Care Med. 2005;6:2-8.

8. Berardi A, Rossi C, Lugli L, et al; GBS Prevention Working Group, EmiliaRomagna. Group B streptococcus late-onset disease: 2003-2010. Pediatrics. 2013;131:e361-e368.

9. Capanna F, Emonet SP, Cherkaoui A, et al. Antibiotic resistance patterns among group B Streptococcus isolates: implications for antibiotic prophylaxis for early-onset neonatal sepsis. Swiss Med Wkly. 2013;143:w13778.

10. Schlapbach LJ, Aebischer M, Adams M, et al; Swiss Neonatal Network and Follow-Up Group. Impact of sepsis on neurodevelopmental outcome in a Swiss National Cohort of extremely premature infants. Pediatrics. 2011;128:e348-e357. 